\title{
Towards A Relational Approach For Tool Creation By Robots
}

\author{
Handy Wicaksono \\ School of Computer Science and Engineering, UNSW Australia \\ handyw@cse.unsw.edu.au
}

\begin{abstract}
Like a human, a robot may benefit from being able to use a tool to solve a complex task. When an appropriate tool is not available, a very useful ability for a robot would be to create a novel one based on its past experience. With the advent of inexpensive $3 \mathrm{D}$ printing, it is now possible to give robots such an ability, at least to create simple tools. We propose a method for learning how to use an object as a tool and, if needed, to design and construct a new tool.
\end{abstract}

\section{Introduction}

Humans use tools to solve complex problems in their daily life. When an appropriate tool is not available, humans can innovate and design a new tool, often based on prior experiences, or even invent it from scratch. In previous work, Brown [2009] has shown how a robot can learn to use an existing object as a tool.

However, tool creation is a much more difficult task, because if the robot has the ability to create objects of arbitrary shape, the space of possible objects available is almost infinite. Thus, tool creation is challenging in giving the learning system the ability to innovate, but not so much that it will never design a useful tool.

Although there is increasing interest in tool use learning, there has been little work in tool creation. Wang et al. [2014] developed a robot that is able to create tools on the fly, but their designs were predefined. One limitation is that most existing work uses a feature-based representation [Mar et al., 2015] which is not expressive enough to be extended to tool creation. A relational representation can overcome this weakness [Brown, 2009].

We introduce a system called CREATIVE (Cognitive Robot Equipped with Autonomous Tool Invention Expertise), which refers to a robot that is able to learn to use a tool and, if needed, design and build a new one. Additionally, unlike Brown [2009], we conduct our experiments using a real Baxter robot, and for testing on large numbers of example, we use its sensor-based simulation. Our main contributions to this research are:

- Developing new representations for describing tools and their functionalities

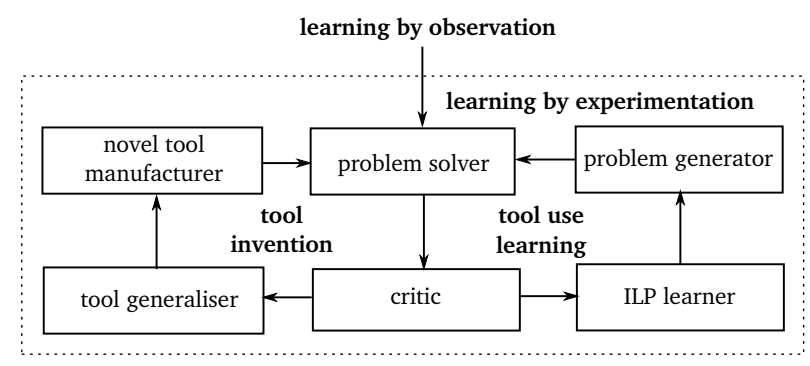

Figure 1: CREATIVE learning framework

- Extending relational learning to build representations for tool creation

- Creating new virtual and physical tools automatically

- Using a physics simulator to minimise real world experiments in action model learning

\section{CREATIVE Mechanism}

The robot is provided with a simple ontology that describes the classification of tools. Each tool has predefined structural properties. The main function of our ontology is to limit the search space when generalisation is performed. It is used as a refinement graph, during learning.

We maintain primitive and abstract states. The latter are represented by Horn clauses to express qualitative relationships between objects. A tool model, as part of a STRIPS action model, captures structural and spatial properties of a tool. It may be provided, in advance, or learned by Inductive Logic Programming (ILP).

There are two main learning stages: learning by observation to acquire an initial action model, and learning by experimentation to refine it. Tool use learning or tool invention is performed, depending on the latest experiment result. We illustrate the learning framework in Fig. 1.

\subsection{Tool Use Learning}

We adopt the learning mechanism proposed by Brown [2009]. Initially, the robot does not have complete action models. The robot is shown a correct example by a trainer, which is used to construct the initial action model via a form of Explanationbased Learning (EBL). A version space [Mitchell, 1977] of action models in updated through learning. 
The system refines the action model by conducting experiments. Positive examples may generalise the most specific hypothesis, then construct an instance that is consistent with its generalisation, and negative examples will do the opposite. This is similar with Golem [Muggleton and Feng, 1990] which performs a relative least general generalisation.

\subsection{Tool Creation}

In tool creation, the refinement graph is used to suggest generalisations by climbing the generalisation hierarchy. The new hypothesis is tested by generating an instance of the hypothesis that does not match previously constructed instances. Even though the refinement graph limits the search of possible generalisations, a reasonably large graph may give rise to many possible configurations.

To avoid manufacturing and testing tools exhaustively, the system uses a simple heuristic. It prioritises new tools that are similar to an existing one, where similarity between representations of the tools is computed by the edit distance, which is the number of edit operations needed to transform one representation into the other. We also conduct the initial experiments in a simulation, and continue in the real world as a validation.

Having the structural properties of new tools, the numerical values for sizes can be acquired by treating the them as constraints for a constraint solver. A tool is "manufactured" by converting the tool's description into a URDF (Universal Robotic Description Format) file, to be spawned in a Gazebo simulation, or into an OpenSCAD file, to be manufactured via a $3 \mathrm{D}$ printer.

Once the potential tools are realised, further learning by experimentation can be performed to validate the usefulness of the novel tool and update the action model. In the ILP framework, learned action models can be stored as background knowledge for future learning.

\section{Results and Future Work}

\subsection{Tool Use Learning Experiment}

We have developed a robotic system capable of learning how to use a hook-like tool in a simulated world and the real world [Wicaksono and Sammut, 2016]. We start with the initial action model using the method of Brown [2009]. Figure 2 shows whole episodes conducted with the real and simulated robot. Sixteen unique episodes are required to learn this tool use task, with most of them are done in simulation. Repeated trials need to be done in the future to obtain statistically meaningful results.

\subsection{Tool Creation Experiment}

We have performed initial experiments of tool creation in simulation. The environment is slightly changed, using a longer tube. This prevents the robot from solving the problem using the old tool and ensures that a new tool with a longer handle is created. Prioritising the tools with smaller edit distances is at least $60 \%$ more efficient than the random selection of tools in a single learning trial. Repeated, and more varied, experiments still have to be done to obtain more convincing results.

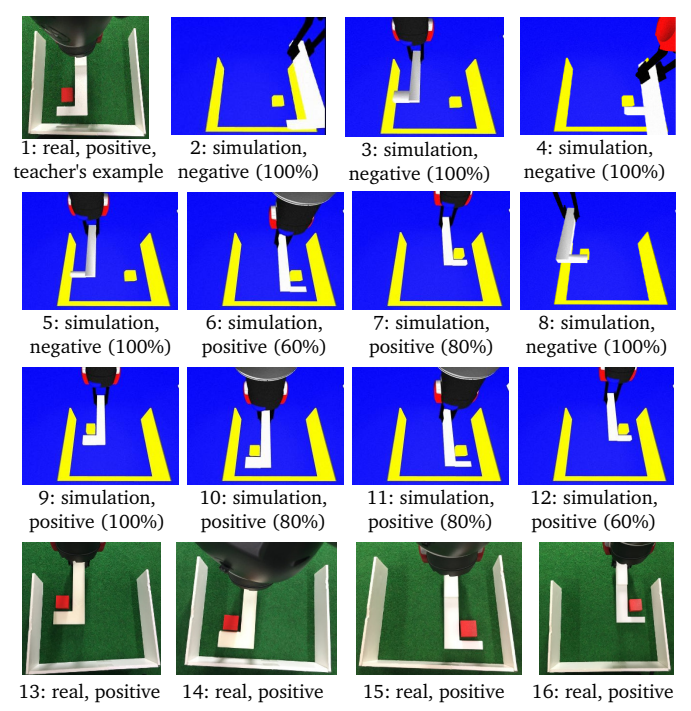

Figure 2: The learning episodes in the real and simulated world. In simulation, each episode is repeated five times and the highest probability result is chosen.

In the future, we aim to perform tool creation in the simulation and real world. The latter is important for validation. A way to measure similarity between a tool model in a simulation and real world must be developed to acquire a final and correct tool model. Tool creation experiments that use different kinds of tools, in addition to a hook-like tool, are also needed to demonstrate the generality and flexibility of our approach.

\section{References}

[Brown, 2009] Solly Brown. A relational approach to tooluse learning in robots. $\mathrm{PhD}$ thesis, School of Computer Science and Engineering, UNSW Australia, 2009.

[Mar et al., 2015] Tanis Mar, Vadim Tikhanoff, Giorgio Metta, and Lorenzo Natale. Self-supervised learning of grasp dependent tool affordances on the icub humanoid robot. In Proceedings of ICRA, May 2015.

[Mitchell, 1977] Tom M Mitchell. Version spaces: A candidate elimination approach to rule learning. In Proceedings of the 5th international joint conference on Artificial intelligence-Volume 1, pages 305-310. Morgan Kaufmann Publishers Inc., 1977.

[Muggleton and Feng, 1990] Stephen Muggleton and Cao Feng. Efficient induction of logic programs. In New Generation Computing. Academic Press, 1990.

[Wang et al., 2014] Liyu Wang, Luzius Brodbeck, and Fumiya Iida. Mechanics and energetics in tool manufacture and use: a synthetic approach. Journal of The Royal Society Interface, 11(100), 2014.

[Wicaksono and Sammut, 2016] Handy Wicaksono and Claude Sammut. Relational tool use learning by a robot in a real and simulated world. In Proceedings of ACRA, December 2016. 